\title{
Babesiosis in Calf: A Case Report
}

\author{
Rajeev Kumar ${ }^{1 *}$ and Shashi Kala ${ }^{2}$ \\ ${ }^{1}$ Class I Veterinary Dispensary Baidyanathpur, Vaishali, Bihar, India \\ ${ }^{2}$ Institute of Animal Health and Production, Bihar, Patna, India \\ *Corresponding author
}

\begin{abstract}
A B S T R A C T
Keywords

Babesiosis, Calf,

Clinical symptoms,

Diagnosis,

Management,

Imidocarb

dipropionate

Article Info

Accepted:

26 June 2018

Available Online:

10 July 2018

Babesiosis is economically the most important arthropod-borne disease of cattle worldwide caused by protozoan parasite of the genus Babesia. Among several species of Babesia, Babesia bigemina and Babesia bovis are most common in tropical and subtropical region. There are age-related immunity to primary infection of cattle with Babesia bovis and Babesia bigemina. Generally young calves exhibit a strong innate immunity in comparison to adult cattle. In this case report, babesiosis was diagnosed in a one month old female calf showed clinical symptoms of anorexia, anaemia, pyrexia, reduced body weight, haemoglobinurea, enlargement of superficial lymph nodes and presence of ectoparasites. Thin blood smear was prepared and examined microscopically by using Geimsa staining technique. The blood smear revealed the presence of piroplasmic organisms of Babesia in RBC. The haematological analysis revealed, reduced haemoglobin concentration $(8.5 \mathrm{gm} / \mathrm{dl})$. The animal was treated with Imidocarb dipropionate at the dose rate of $1 \mathrm{ml} / 100 \mathrm{~kg}$ body weight with supportive therapy. In order to control babesiosis owner was advised to use acaricides and also advised for rotation of drugs and to administer proper dosage to prevent acaricidal resistant.
\end{abstract}

\section{Introduction}

Babesiosis is a haemoprotozoan disease caused by genus Babesia coming under family Babesidae and order Piroplasmida, within the phylum Apicomplexa. It is one of the common tick borne haemoprotozoan disease, affects a wide range of domestic and wild animals. The infection has long been recognised as an economically important disease of cattle, horses, and dogs and has gained increasing attention as an emerging zoonotic disease (Maharana et al., 2016). There are several species of Babesia, out of which Babesia bigemina and Babesia bovis are most common in tropical and subtropical region. Bovine babesiosis is principally maintained by sub clinically infected cattle that have recovered from disease.

The morbidity and mortality are influenced by prevailing treatments employed in an areas and previous exposure to the infection. Walker and Edward (1927), first time reported babesiosis in India. McLeod and Kristjanson (1999), reported the annual loss of 57.2 
million US dollars due to babesiosis in India. The organism is intraerythrocytic protozoan parasites, multiply asexually in the erythrocytes of vertebrate host producing 2, 4 or more trophozoites. Trophozoites liberated from infected red blood cells and invade new cells and it infects large number of red blood cells (Soulsby, 1882).

B. bigemina and B. Bovis are mainly transmitted by one host tick Boophilus spp, in which transovarian transmission occurs. Basesiosis also transmitted mechanically by insects or during surgical procedure castration, dehorning etc vide surgical instruments. Stage to stage transmission also performed by 2 or 3 host ticks. Clinical symptoms are characteristic of increased body temperature, increased pulse rate, anorexia, and in acute infection haemoglobinurea and haemolytic anaemia but the symptoms vary according to the species of parasites and host factors (i.e. age, immune status).

The calves up to 9-12 months of age are generally resistant due to inverse age resistance, but the clinical symptoms of babesiosis in neonatal calves were inability to suckling, high fever, coffee colour urine, jaundice and deep shallow respiration (Karunakaran et al., 2011; Venu et al., 2013; Bal et al., 2016). In endemic areas, cattle become infected at young age and develop a long-term immunity. Infected animals develop a lifelong immunity against re-infection with the same species. However, cross protection is also evident in Babesia bigemina.

Hence the present case report of babesiosis in female calf was conducted to study the incidence of disease in calves.

\section{Case history and clinical observation}

A one month old female calf (Figure 1) was presented to the veterinary dispensary with a history of anorexia, anaemia, pyrexia, reduced body weight, haemoglobinurea, enlargement of superficial lymph nodes and presence of ectoparasites. The calf was clinically examined and recorded pale mucous membrane, increased rectal temperature $\left(103.5{ }^{\circ} \mathrm{F}\right)$ and presence of ectoparasites. Blood sample was collected from jugular vein in a vial containing anticoagulant (EDTA vial) for haemoprotozoan examination and haematology. Faecal sample was also collected for screening of G.I. parasites.

\section{Laboratory examination}

Faecal sample was examined microscopically to screen out the G.I. parasites by direct method as well as sedimentation and floatation method. For haemoprotozoan test a thin blood smear was prepared, air dried, fixed with methanol and stained with Giemsa stain (1:10 ratio) for 30-40 minutes and then extra stain was poured off and removed by washing with tap water. The stained smear was examined under oil immersion lens (100X). The parasite was identified according to the characters described by Soulsby (1982). Further for haematology haemoglobin estimation was performed.

\section{Results and Discussion}

By using Geimsa staining technique, stained thin blood smear revealed the presence of piroplasmic organisms of Babesia in RBC (Figure 2). The haematological analysis revealed, reduced haemoglobin concentration (8.5 gm/dl). The animal was treated with Imidocarb dipropionate at the dose rate of $1 \mathrm{ml} / 100 \mathrm{~kg}$ body weight and Ivermectin at the dose rate $0.2 \mathrm{mg} / \mathrm{kg}$ SC. Imidocarb dipropionate is an antiprotozoal drug for the treatment and prevention of babesiosis in cattle, sheep, horses and dogs. Ivermectin was used for the control of endo and ectoparasites. The calf was also treated with multivitamin as 
a supplement of iron and vitamin to increase red blood cells formations and stomachic powder to increase appetite. After one week, blood smear was again examined by using Geimsa staining technique and was found negative for babesiosis. Haematology revealed within the normal range. The colour of urine

Fig.1

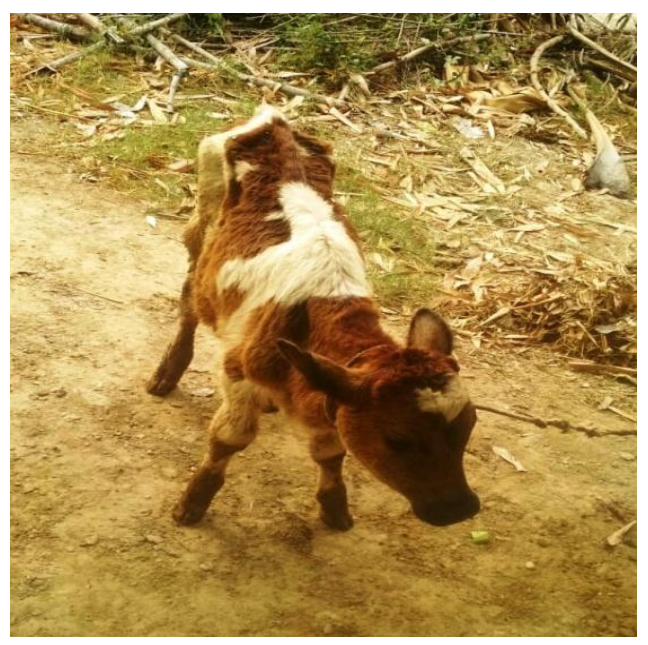

Venu et al., (2013) also diagnosed and reported Babesia bigemina infection in a 14day old Jersey female calf showing clinical symptoms of high fever, increased respiratory rate, pale mucous membrane and haemoglobinuria.

However, Zintl et al., (2005) observed that the calves infected with the tick-borne parasites Babesia spp. do not develop severe clinical babesiosis and instead they display persistent low parasitaemia without any apparent ill-effects. Bal et al., (2016) treated babesiosis affected animals successfully with diminazene aceturate, hematinics and antipyretics.

Imidocarb dipropionate is the drug of choice to treat bovine babesiosis followed by Dimenazeneaceturate with $100 \%$ and $90 \%$ efficacy at 10 day post treatment respectively (Ukwueze and Orajaka, 2014). Currently was found normal. Karunakaran et al., (2011) and Ukwueze and Orajaka (2014) successfully treated a two months old calf and a twenty days old calf respectively with a single dose of diminazeneaceturate along with supportive therapy.

Fig.2

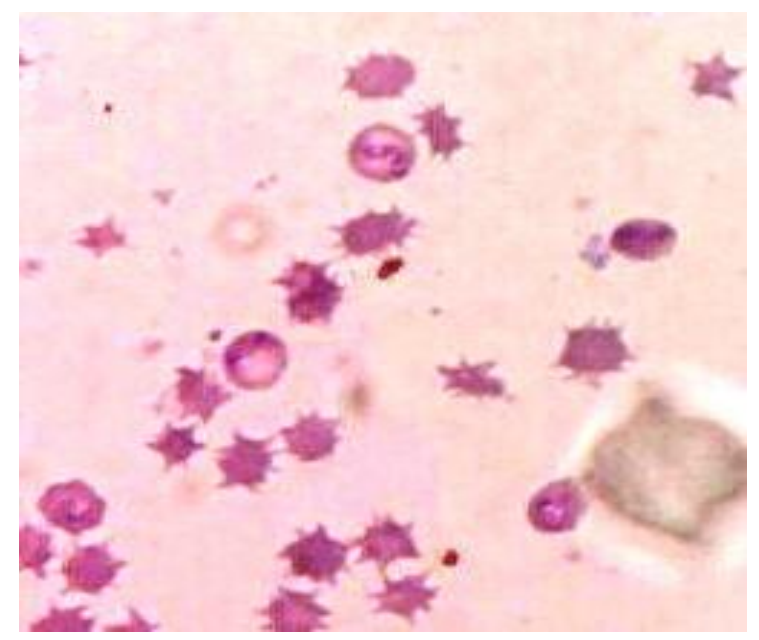

these two drugs are most widely used as babesiacides. Treatment with long-acting oxytetracycline following vaccination significantly reduces parasitaemia and red blood cell destruction without inhibiting the development of immunity.

In order to control babesiosis owner was advised to use acaricides and also advised for rotation of drugs and to administer proper dosage to prevent acaricidal resistant. The common acaricides used are chlorinated hydrocarbons, carbamates, natural and synthetic pyrethrins and ivermectins. Live attenuated strains of $B$. bovis, B. bigemina, $B$. divergens are used to vaccinate cattle.

\section{References}

Bal, M.S., Mahajan, V., Filia, G., Kaur, P. and Singh, A. (2016) Diagnosis and management of bovine babesiosis 
outbreaks in cattle in Punjab state. Veter. World, (9):1370-1374.

Karunakaran, S., Pillai, U., Narayana, K.A.M., Aswathy, G., Rajimon, K.T. and Sumangala, M. (2011) Babesia bigemina infection in a twenty day old calf. J. Indian Vet. Assoc. Kerala, 9: 49-50.

Maharana B.R., Tewari A.K., Saravanan B.C., Sudhakar, N.R. (2016) Important hemoprotozoan diseases of livestock: Challenges in current diagnostics and therapeutics: An update, Veterinary World, 9(5): 487-495.

McLeod, R. and Kristjanson, P. (1999) Final report of joint esys/ILRI/ACIAR Tick Cost project- Economic impact of ticks and TBD to livestock in Africa, Asia and Australia. International Livestock Research Institute (ILRI), Nairobi, Kenya.

Soulsby, E.J.L. (1982) Helminths, arthropods and protozoan of domesticated animals, $7^{\text {th }}$ edn., Bailiere Tindall and Cassell Ltd., London.

Ukwueze, C.S and Orajaka, C.F. (2014) Babesiosis in a calf: A case report. IOSR J. Agri. And Vet. Sci., 7(11) Ver. II; 72-74.

Venu, R., Sailaja, N., Rao, K.S., Jayasree, N. and Prasad, W.L.N. (2013) Babesia bigemina infection in a 14 day old Jersey crossbred calf: A case report $J$. Parasit.Dis. DOI:10.1007/s12639013-0338-x

Walker,G.K. and Edward,J.T.(1927) Some Diseases of Cattle in India. Government of India, Calcutta. p29.

Zintl, A., Gray, J.S., Skerrett, H.E. and Mulcahy, G. (2005) Possible mechanisms underlying age-related resistance to bovine babesiosis. Parasite Immunology, 27:115-120.

\section{How to cite this article:}

Rajeev Kumar and Shashi Kala. 2018. Babesiosis in Calf: A Case Report. Int.J.Curr.Microbiol.App.Sci. 7(07): 3951-3954. doi: https://doi.org/10.20546/ijcmas.2018.707.459 